\title{
Genome-wide association analysis reveals quantitative trait loci and candidate genes involved in yield components under multiple field environments in cotton (Gossypium hirsutum)
}

Guozhong Zhu ${ }^{1+}$, Sen Hou ${ }^{1+}$, Xiaohui Song ${ }^{1+}$, Xing Wang ${ }^{1}$, Wei Wang ${ }^{2}$, Quanjia Chen ${ }^{3}$ and Wangzhen Guo ${ }^{{ }^{*}}$

\begin{abstract}
Background: Numerous quantitative trait loci (QTLs) and candidate genes associated with yield-related traits have been identified in cotton by genome-wide association study (GWAS) analysis. However, most of the phenotypic data were from a single or few environments, and the stable loci remained to be validated under multiple field environments.

Results: Here, 242 upland cotton accessions collected from different origins were continuously investigated for phenotypic data of four main yield components, including boll weight (BW) and lint percentage (LP) under 13 field environments, and boll number per plant (BN) and seed index (SI) under 11 environments. Correlation analysis revealed a positive correlation between BN and LP, BW and SI, while SI had a negative correlation with LP and BN. Genetic analysis indicated that LP had the highest heritability estimates of $94.97 \%$, followed by $92.08 \%$ for $\mathrm{SI}, 86.09 \%$ for BW, and $72.92 \%$ for BN, indicating LP and SI were more suitable traits for genetic improvement. Based on 56,010 highquality single nucleotide polymorphisms (SNPs) and GWAS analysis, a total of 95 non-redundant QTLs were identified, including 12 of BN, 23 of BW, 45 of LP, and 33 of SI, respectively. Of them, 10 pairs of homologous QTLs were detected between $\mathrm{A}$ and $\mathrm{D}$ sub-genomes. We also found that 15 co-located QTLs with more than two traits and 12 high-confidence QTLs were detected under more than six environments, respectively. Further, two NET genes (GH_A08G0716 and GH_A08G0783), located in a novel QTL hotspot (qt|24, qt125 and qlt26) were predominately expressed in early fiber development stages, exhibited significant correlation with LP and SI. The GH_A07G1389 in the stable qt119 region encoded a tetratricopeptide repeat (TPR)-like superfamily protein and was a homologous gene involved in short fiber mutant ligon lintless-y $\left(L_{y}\right)$, implying important roles in cotton yield.
\end{abstract}

\footnotetext{
*Correspondence: moelab@njau.edu.cn

${ }^{\dagger}$ Guozhong Zhu, Sen Hou and Xiaohui Song contributed equally to this work.

' State Key Laboratory of Crop Genetics and Germplasm Enhancement, Cotton Germplasm Enhancement and Application Engineering Research Center (Ministry of Education), Nanjing Agricultural University, Nanjing 210095, China

Full list of author information is available at the end of the article
}

(C) The Author(s) 2021. Open Access This article is licensed under a Creative Commons Attribution 4.0 International License, which permits use, sharing, adaptation, distribution and reproduction in any medium or format, as long as you give appropriate credit to the original author(s) and the source, provide a link to the Creative Commons licence, and indicate if changes were made. The images or other third party material in this article are included in the article's Creative Commons licence, unless indicated otherwise in a credit line to the material. If material is not included in the article's Creative Commons licence and your intended use is not permitted by statutory regulation or exceeds the permitted use, you will need to obtain permission directly from the copyright holder. To view a copy of this licence, visit http://creativecommons.org/licenses/by/4.0/. The Creative Commons Public Domain Dedication waiver (http://creativeco mmons.org/publicdomain/zero/1.0/) applies to the data made available in this article, unless otherwise stated in a credit line to the data. 
Conclusions: The present study provides a foundation for understanding the regulatory mechanisms of yield components and may enhance yield improvement through molecular breeding in cotton.

Keywords: Gossypium hirsutum, Yield components, Genome-wide association study, Quantitative trait loci, Multiple field environments

\section{Background}

Cotton is an important commercial crop that provides the most natural fiber globally and is also an important source of edible oil. Of the cultivated cotton species, upland cotton (Gossypium hirsutum L.) contributes more than $95 \%$ of total cotton production due to its high yield and wide adaptability [1]. Developing high-yielding varieties has been one of the essential targets in cotton breeding. However, the improvement of cotton yield via conventional breeding programs remains low and slow because the narrow genetic background of upland cotton has resulted in breeding bottlenecks [2]. Hence, it is of great significance to explore and pyramid the elite quantitative trait loci (QTLs)/genes related to yield components for improving cotton yield through molecular breeding.

The yield components of cotton mainly include boll number per plant $(\mathrm{BN})$, boll weight $(\mathrm{BW})$, lint percentage (LP), seed index (SI), and lint index (LI), which are quantitatively inherited and are easily influenced by the environment [3]. Several QTLs for cotton yield-related traits have been identified using molecular markers and bi-parental linkage mapping analysis $[4,5]$. However, it is challenging to exploit QTLs through markers assisted breeding due to the limited number of markers and the large QTL regions. With the rapid development of highthroughput sequencing technologies and statistical methods, the genetic basis of cotton yield-related traits has been preliminarily revealed. In recent five years, the assembly and improvement of tetraploid cultivated cotton genome significantly accelerated the mapping of genes for the important traits in cotton [6-11]. Based on the reference genome sequence, a large number of QTLs and candidate genes associated with yield-related traits were identified by genome-wide association study (GWAS) analysis [12-15]. The power of genome-wide association analysis is mainly based on four factors: the richness of genetic diversity, the veracity of trait acquisition, marker density, and statistical methods [16]. In most previous studies, due to the experimental design of a single environment and the single-locus GWAS approaches, many stable loci remain to be detected. Multi-environment and multi-locus GWAS coupled with improved experimental design and associated methods may increase efficiency to mine QTLs/genes related to fiber yield traits, which is still challenging in cotton breeding.
In the present study, 242 upland cotton accessions with diverse origins were planted in multiple environments over the years for phenotyping investigation of four main yield components, BW and LP under 13 natural environments, and BN and SI under 11 environments. GWAS analysis was conducted based on a multi-locus random-SNP-effect mixed linear model, and stable QTLs associated with yield components were revealed in multiple environments. Combined with transcriptome analysis, the expression patterns of candidate genes were investigated, and key genes contributing to cotton yield were predicted. The results may be helpful to understand the genetic architecture of yield traits better and provide molecular markers and candidate genes for designing high-yielding cotton lines via molecular breeding.

\section{Results}

Phenotypic variation of the four yield-related traits

We analyzed the phenotypic data of four yield-related traits boll number $(\mathrm{BN})$, boll weight $(\mathrm{BW})$, lint percentage (LP), and seed index (SI) in multiple field environments to evaluate the phenotypic variation in the natural population of 242 upland cotton accessions (Additional file 1 Table S1). BN, BW, LP, and SI differed significantly from 1.7 to $35.5,2.5 \mathrm{~g}$ to $9.1 \mathrm{~g}, 23.0 \%$ to $50.2 \%$, and $6.7 \mathrm{~g}$ to $17.1 \mathrm{~g}$, respectively (Additional file 2 Table S2). BN exhibited the largest coefficient of variation $(\mathrm{CV})$, ranging from $13.13 \%$ to $24.26 \%$, while LP showed the smallest CV ranging from $7.19 \%$ to $10.53 \%$. The best linear unbiased prediction (BLUP) across multiple environments estimates showed that the phenotypic value of $\mathrm{BN}, \mathrm{BW}$, LP and SI from BLUP ranged from 9.3 to $15.3,4.6 \mathrm{~g}$ to $6.3 \mathrm{~g}, 31.7 \%$ to $45.8 \%$, and $8.7 \mathrm{~g}$ to $12.3 \mathrm{~g}$, respectively (Additional file 2 Table S2, Additional file 3 Fig. S1). The broad-sense heritability $\left(h^{2}\right)$ of each trait was estimated based on multi-environment phenotypic data (Table 1) to explore the breeding potential of the traits. The high heritability estimates were found for LP $(94.97 \%)$ and SI (92.08\%), followed by BW (86.09\%). However, the heritability of $\mathrm{BN}(72.92 \%)$ was the lowest, indicating the BN was inclined to be influenced by environments. The analysis of variance (ANOVA) was performed to investigate the effects of genotype $(\mathrm{G})$, environment $(\mathrm{E})$, and $\mathrm{G} \times \mathrm{E}$ interactions among the four traits in multi-environment (Table 1). The results indicated that these traits were also 
Table 1 The analysis of variance and broad heritability of four yield-related traits

\begin{tabular}{|c|c|c|c|c|c|c|}
\hline \multirow[t]{3}{*}{ Trait } & Factors & Df & Sum-sq & Mean-sq & $F$ & $h^{2}(\%)$ \\
\hline & Env & 10 & $199,757.98$ & $19,975.80$ & $4137.62^{* * *}$ & \\
\hline & Rep(Env) & 11 & 858.47 & 78.04 & $16.17^{* * *}$ & \\
\hline \multirow[t]{5}{*}{ BN } & Geno & 241 & 8938.48 & 37.09 & $7.68^{* * *}$ & 72.92 \\
\hline & Geno $\times$ Env & 2405 & $24,123.73$ & 10.03 & $2.08^{* * *}$ & \\
\hline & Error & 2643 & $12,760.00$ & 4.83 & & \\
\hline & Env & 12 & 1733.65 & 144.47 & $717.61^{* * *}$ & \\
\hline & Rep(Env) & 13 & 62.04 & 4.77 & $23.7^{* * *}$ & \\
\hline \multirow[t]{5}{*}{ BW } & Geno & 241 & 880.89 & 3.66 & $18.16^{* * *}$ & 86.09 \\
\hline & Geno $\times$ Env & 2886 & 1475.69 & 0.51 & $2.54^{* * *}$ & \\
\hline & Error & 3111 & 626.31 & 0.20 & & \\
\hline & Env & 12 & $28,466.88$ & 2372.24 & $899.41^{* * *}$ & \\
\hline & Rep(Env) & 13 & 112.00 & 8.62 & $3.27^{* * *}$ & \\
\hline \multirow[t]{5}{*}{ LP } & Geno & 241 & $39,670.63$ & 164.61 & $62.41^{* * *}$ & 94.97 \\
\hline & Geno $\times$ Env & 2887 & $24,035.90$ & 8.33 & $3.16^{* * *}$ & \\
\hline & Error & 3108 & 8197.54 & 2.64 & & \\
\hline & Env & 10 & 3020.86 & 302.09 & $892.59^{* * *}$ & \\
\hline & Rep(Env) & 11 & 21.45 & 1.95 & $5.76^{* * *}$ & \\
\hline \multirow[t]{3}{*}{ SI } & Geno & 241 & 2966.70 & 12.31 & $36.37^{* * *}$ & 92.08 \\
\hline & Geno $\times$ Env & 2409 & 2358.90 & 0.98 & $2.89^{* * *}$ & \\
\hline & Error & 2633 & 891.11 & 0.34 & & \\
\hline
\end{tabular}

BN: boll number per plant; BW: boll weight; LP: lint percentage; SI: seed index; Env: environment; Rep: replication; Geno: genotype; *** indicated P value at the 0.0001 levels

significantly subjected to genotypes and environments interactions. Correlation analysis showed a positive correlation between BN and LP, while SI showed a positive correlation with $\mathrm{BW}$ and a negative correlation with $\mathrm{BN}$ and LP (Fig. 1).

\section{Genome-wide association analysis}

To explore the genetic factors associated with the four yield-related traits, we conducted a GWAS analysis using the genotypic data of 56,010 high-quality SNPs ([17], Additional file 4 Table S3) and the phenotypic data of the four yield-related traits in multiple environments and the BLUP. In total, 560 quantitative trait nucleotides (QTNs) were identified as loci significantly associated with the four traits. With $200 \mathrm{~kb}$ as the linkage disequilibrium (LD) threshold for merging QTNs into the same QTL, a total of 360 QTLs of four traits were identified from all environments and BLUP (Additional file 5 Table S4). Further, QTLs consistent across at least two environments were declared as stable QTLs; thus, 95 candidate QTLs were identified with 12 of BN, 23 of BW, 45 of LP and 33 of SI (Fig. 2 and Additional file 6 Table S5). Of them, 38 co-located with previous studies (Additional file 6 Table S5), and 57 were novel and were reported for the first time in the current study. Furthermore, 10 pairs of homologous QTLs were detected between A and D sub-genomes (Additional file 7 Table S6), indicating a common selection of two sub-genomes to improve yield components in upland cotton.

There were 15 QTLs detected simultaneously to be associated with two or more traits (Additional file 6 Table S5, Additional file 8 Fig. S2). For example, nine common QTLs for LP and SI were identified, consistent with the high correlation $(R=-0.58)$ between the two traits. These candidate QTLs were widely distributed on the 26 chromosomes with a little more on At sub-genome comparing to Dt sub-genome (Additional file 9 Fig. S3).

The QTLs consistently detected in more than six environments were defined as high-confidence QTLs; thus, 12 high-confidence QTLs were identified (Fig. 2, Additional file 6 Table S5). For example, qtl24 was associated with LP in seven environments and qtl19 with SI in six environments. Furthermore, three QTLs were found to be associated with three traits simultaneously: qtl48 were identified to co-locate with BW, BN, and LP in six environments; qt161 and qtl83 with BW, SI, and LP in nine environments, implying the genetic stability of these QTLs for yield components in upland cotton.

\section{Identification of candidate genes in yield-related QTL regions}

Genes located in the candidate QTL regions and with an expression of more than 3 transcripts per million (TPM) in cotton tissues were extracted based on the 


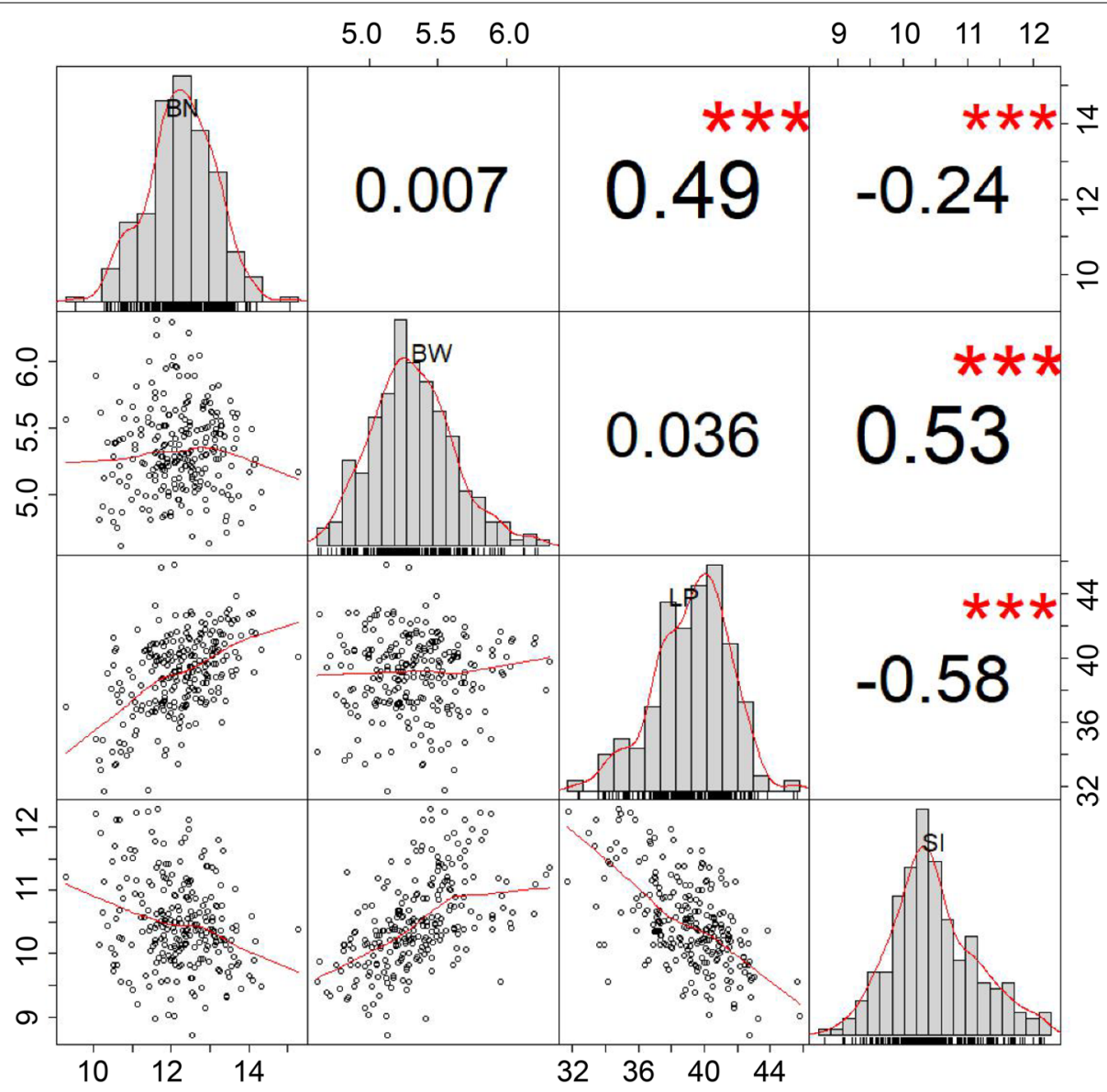

Fig. 1 Correlation analysis of four yield-related traits. The number in these boxes indicated correlation coefficient ( $R$ value). The correlation coefficient and the significance between traits were conducted using BLUP. *** indicated $P$ value at the 0.001 levels. BN: boll number per plant; BW: boll weight; LP: lint percentage; Sl: seed index

released G. hirsutum TM-1 genome [11]. A total of 4144 genes were located in the candidate QTL regions, with 547 related to BN, 1096 to BW, 2236 to LP and 1430 to SI. By filtering the low expressed genes, 1490 candidate genes were identified, with 347 related to BN, 407 to BW, 606 to LP and 424 to SI (Additional file 10 Table S7). Gene Ontology (GO) analysis for each trait was individually conducted to investigate the candidate genes' function (Fig. 3, Additional file 11 Table S8). We found that candidate genes from the QTLs region of different traits were predominately enriched in different biological processes. For LP, the function of genes was mainly enriched in glycol-metabolism-related processes, such as single-organism carbohydrate metabolic process, glucose metabolic process, carbohydrate biosynthetic process. Besides, other processes closely related to fiber development were enriched, such as microtubule-based process, thylakoid membrane organization, regulation of plant epidermal cell differentiation, and plant-type secondary cell wall biogenesis. For SI, embryonic development and flowering-related processes were preferentially enriched, such as positive regulation of post-embryonic development, long-day photoperiodism, flowering, and vegetative to the reproductive phase transition meristem. For BW, the candidate genes involved in energy metabolism, including long-chain fatty acid biosynthetic process, glycolytic process, ADP metabolic process and single-organism carbohydrate catabolic process. For BN, the enriched processes contained tryptophan catabolic process, cell cycle G1/S phase transition and floral organ abscission. In general, these predominately enriched biological processes from QTL regions of each trait were highly related to the developmental process of these yield components.

\section{Key QTLs and genes associated with yield-related traits}

Among 12 high-confidence QTLs, we found a QTL hotspot (A08: 10.7-14.6 Mb) containing three novel QTLs, qtl24, qtl25 and qlt26 for LP, SI and LP, respectively (Fig. 4a). Via tissue and organ transcriptome profiling, we found that 15 genes located on the stable QTL 

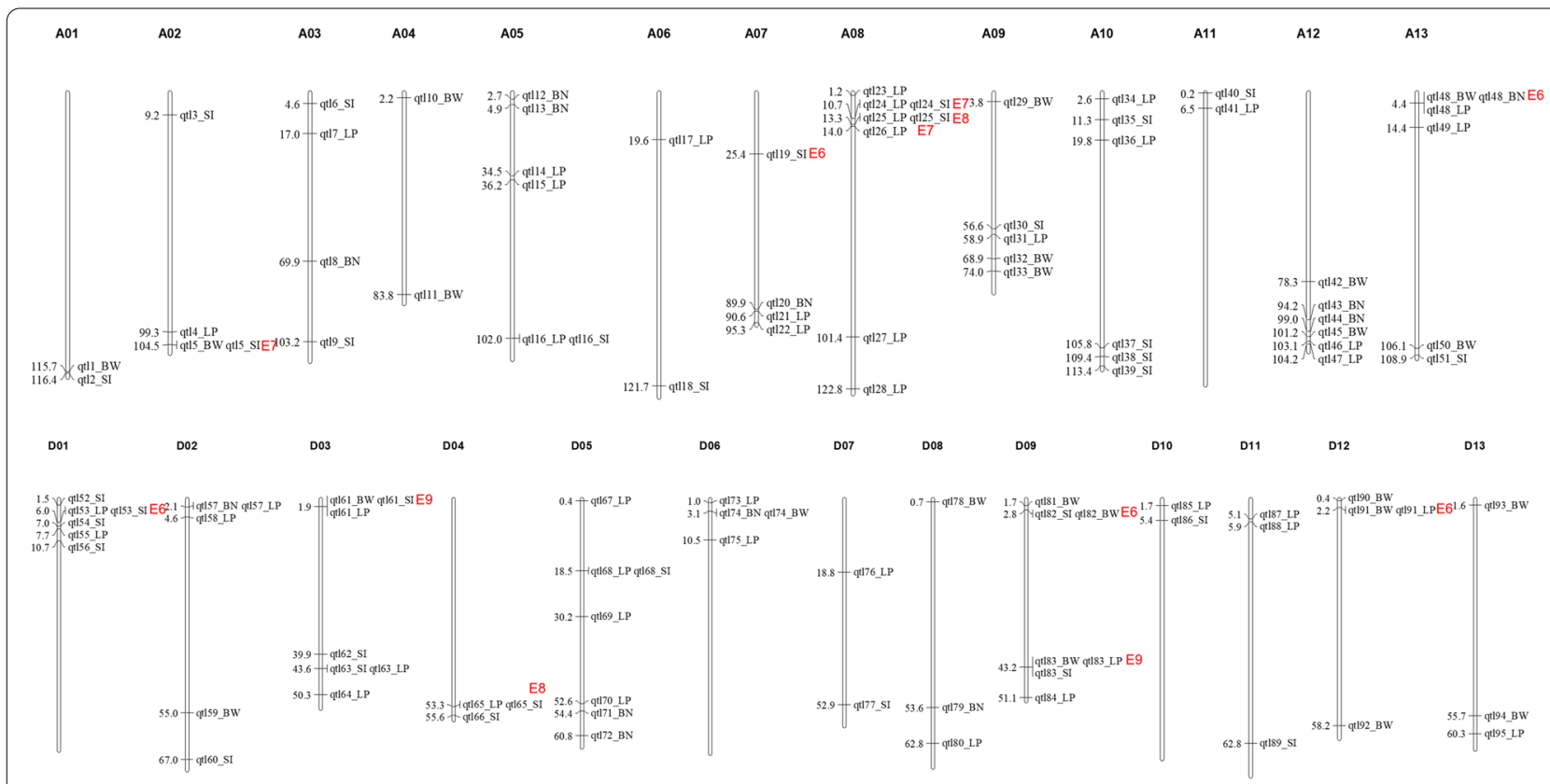

Fig. 2 Distributions of candidate QTLs associated with four yield-related traits. The numbers on the left of chromosomes indicates physical distance of the corresponding QTLs. The red character indicates high-confidence QTLs with detected times in different environments (E)

hotspot were predominantly expressed in ovule or fiber during fiber development (Fig. 4b). Of them, two encoding kinases interacting (KIP1-like) family protein genes (GH_A08G0716 and GH_A08G0783), its' orthologous genes encoding plant-specific Networked (NET) superfamily of actin-binding proteins in Arabidopsis, were identified in qtl24 and qtl25, respectively. The two genes were highly expressed in fiber development, especially at the early fiber development stages (Fig. 4c). Further, two QTNs (TM22408 and TM22482) which were closest to the two NET genes, respectively, were selected to investigate the correlation between genes and yield components. By comparing the phenotypic difference of the two genotypes (Fig. 4d), the LP values with a G genotype were significantly higher than that with an A genotype in QTN TM22408, while the SI values were lower with a G genotype. In QTN TM22482, a T genotype showed higher LP values and lower SI values than an A genotype. The results indicated that these two genes have potential functions in increasing LP. We also found two genes qt124: GH_A08G0734 and qtl26: GH_A08G0793, highly expressed at 0 DPA and 25 DPA of fiber development, respectively. GH_A08G0734 encoded a protein phosphatase $2 \mathrm{~A}$ (PP2A) regulatory $\mathrm{B}$ subunit family protein to regulate the brassinosteroid-mediated signaling pathway [18]. GH_A08G0793 encoded a bidirectional sugar transporter SWEET12-like protein, which also plays a crucial role in fiber development [19].
Cotton fiber and seed development are equally important because of the specialized hair-like fibers derived from the seed-coat epidermis. Of the stable QTLs, most were simultaneously associated with LP and SI. We found that most candidate genes were highly expressed in ovule at the early stages of fiber development through transcription profile analysis, indicating that SI plays a vital role in fiber yield. A qtl19 was identified to be associated with SI and 17 candidate genes on chromosome A07 in the region of $25.4-26.5 \mathrm{Mb}$. Of the genes, most were highly expressed from -3 DPA to 1 DPA during fiber development (Additional file 12 Fig. S4a). GH_A07G1389 encoded a tetratricopeptide repeat (TPR)-like superfamily protein, previously reported to be related to Ligonlintless mutant phenotype, and was highly expressed from -3 DPA to 1 DPA during fiber development (Additional file 12 Fig. 4b). With the closest QTN TM19889, the LP values with a $G$ genotype were significantly higher than that with an A genotype, while the SI values were lower with a G genotype (Additional file 12 Fig. S4c). We also found that a qtl83 (D09:43.2-44.0 Mb), which was detected in nine different environments, was simultaneously associated with BW, LP and SI. Within the qtl83 region, 28 candidate genes were identified, and most of them were involved in fiber development, such as $G H_{-}$ D09G1670 (EXPA4), GH_D09G1688 (AGD7) and GH_ D09G1611 (ARF16), indicating their important roles in yield components formation. 


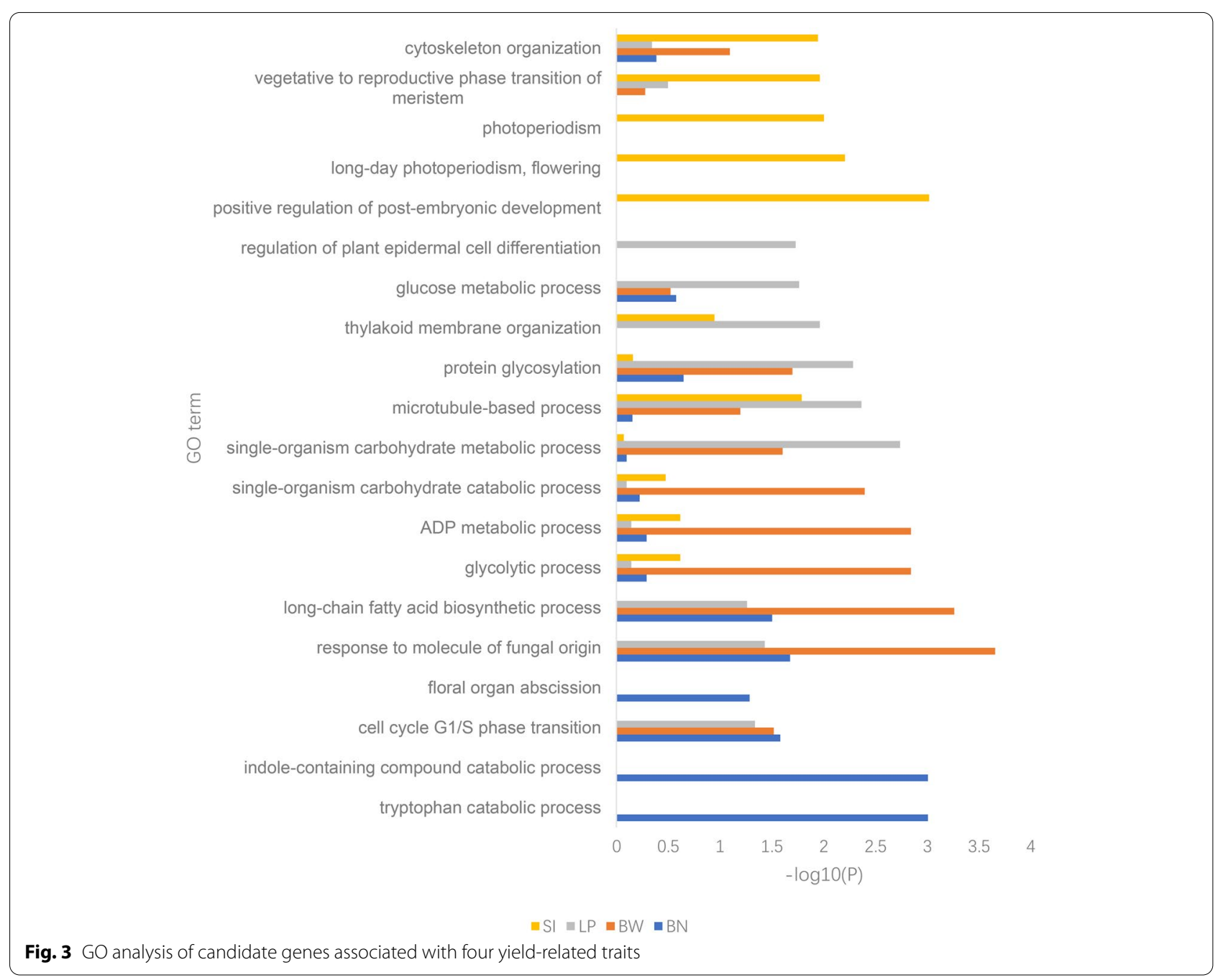

\section{Discussion}

Improving yield is the first breeding objective in crop breeding practices. Cotton is an important economic crop, and its yield components mainly include boll number, boll weight, lint percentage, seed index, lint index etc. It is of great significance to discover QTLs and candidate genes related to yield traits for genetic improvement of cotton to develop high-yielding cultivars. With the release of genome-wide sequence information, lots of QTLs and genes associated with cotton yield traits were identified by GWAS analysis [12, 20-22]. Nevertheless, the yield components are complex quantitative traits controlled by multiple genes and affected by environments. Most GWAS methods are based on a fixed-SNP-effect mixed linear model (MLM) and single-marker analysis, requiring strict correction for the $P$ values and containing many minor-effect QTLs. Previous studies using the multi-locus mixed linear model have also indicated that the model can improve the power and robustness of association analysis [23]. In this study, 242 upland cotton accessions with different origins were planted and investigated under multiple field environments over the years. Through GWAS analysis with the multi-locus mixed linear model, stable QTLs and candidate genes for the four main yield components were systematically revealed.

$\mathrm{BN}$ is a major yield component that significantly contributes to the individual plant yield of cotton [22]. In a previous study, we have also found that $\mathrm{BN}$ is the most important factor in cotton fiber yield [20]. However, the high $\mathrm{CV}$ value and low heritability of $\mathrm{BN}(72.92 \%)$ indicate that the trait is greatly influenced by the environment and is difficult to be controlled in genetic improvement. The BW of cotton is a complex trait affected by seed weight and fiber weight per boll; therefore, SI and LP are two important traits in cotton yield improvement. In this study, LP and SI showed high heritability of $94.97 \%$ and $92.08 \%$, respectively, similar to previous reports $[12,16]$. 

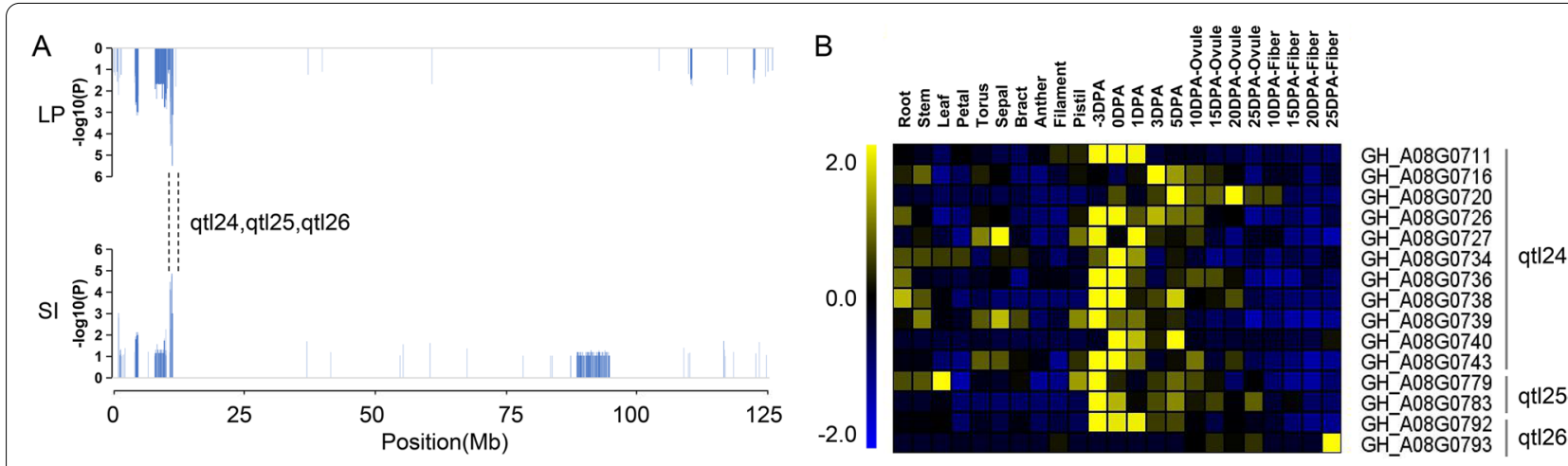

C
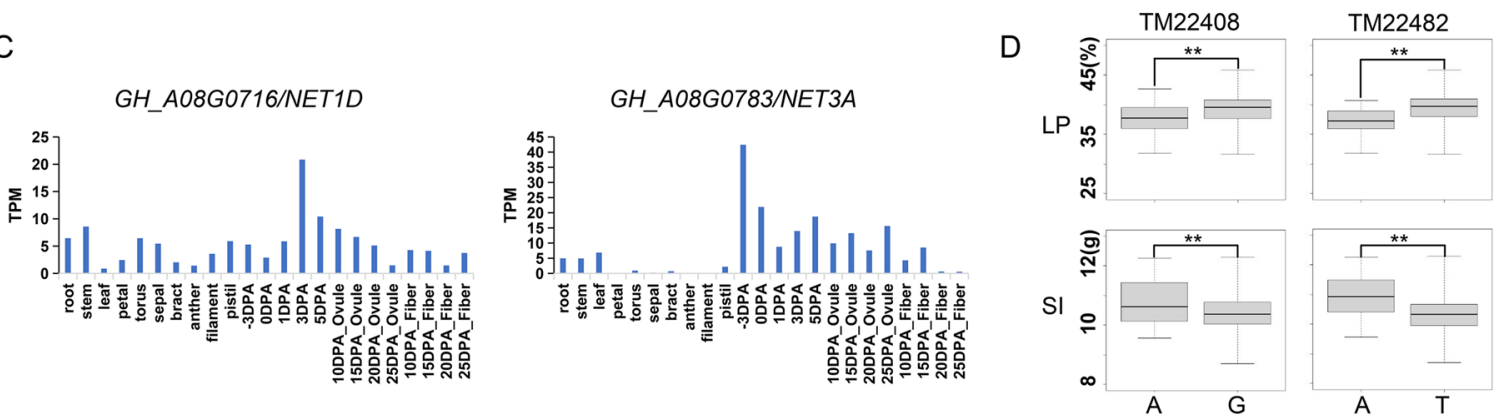

Fig. 4 QTLs and candidate genes located on a QTL hotspot on chromosome A08. a. The QTL hotspot associated with LP and SI on chromosome A08. b. Expression heatmap of candidate genes in the three stable QTLs. c. The expression patterns of two NET genes in different tissues. d. Box plots for the phenotypic values of QTN closest to the two NET genes

Furthermore, a significant negative correlation was identified between LP and SI, and many co-located QTLs were identified both for LP and SI [12, 22]. At present, most of the studies focus on genetic improvement of LP $[12,21]$, while SI is relatively few. We found that there was potential to improve fiber yield through phenotypic analysis by balancing SI and LP values of cotton. As the carrier of fiber, the ovule plays an essential role in fiber development [24]. Manipulating some of the candidate genes identified in fiber cells has yielded no effect or only a marginally positive effect on fiber yield or quality [25], implying the synergistic effect between ovule and fiber development in contributing cotton yield.

We found that many fiber development candidate genes were highly expressed in ovules. During the evolution and domestication of cotton, cotton fiber yield has been strongly correlated with increased seed size [26, 27]. However, the negative correlation between SI and LP indicated that the small seed was conducive to increasing cotton fiber yield for modern cultivars. Therefore, we speculate that a suitable range of seed size effectively increases cotton fiber yield and keeps seed vigor. The regulation of ovule on fiber development could be further explored to identify candidate genes for fiber yield improvement.

The previous studies have detected a large number of associated loci for fiber yield via GWAS analysis.
However, the yield traits are quantitatively inherited and easily affected by environmental factors [28]. Thus, most detected QTLs are difficult to confirm and limited in use. In the present study, 560 QTNs were identified. However, several QTNs had minimal QTN effect and almost zero of $r^{2}$, which might be false positive from the software algorithm and were useless for breeding. We also found that these QTNs with low $r^{2}$ values were associated only in a single environment. Therefore, to improve the authenticity and validity of QTLs, we selected QTLs detected in two or more environments as candidate QTLs. In the 95 candidate QTLs, 38 co-located QTLs have been identified in multiple reported studies [12, 13, 15, 20, 21, 23, 29, 30]. In addition, 57 novel QTLs were detected in multiple environments; for example, qtl83 was associated with $\mathrm{BW}, \mathrm{LP}$ and SI in nine environments, providing more loci and markers for genetic improvement of cotton yield traits. We also found that most of the candidate QTLs were trait-specific, implying the independent regulation mechanism among different yield components. However, 15 QTLs were identified simultaneously in multiple traits and contributed pleiotropically to the improvement of cotton yield through marker-assisted breeding.

To identify key genes suitable for breeding utilization of cotton, we selected the 12 high-confidence QTLs detected in multiple environments for further analysis. 
In these QTLs, a QTL hotspot contained three novel QTLs (qtl24, qtl25 and qlt26) on chromosome A08 was detected. The qt125 explained the highest phenotypic variation with $26.49 \%$ for LP and $20.25 \%$ for SI. In the QTL hotspot, 15 candidate genes were identified, and two genes (GH_A08G0716 and GH_A08G0783) encoding the NET superfamily proteins, which potentially couples different membranes to the actin cytoskeleton in plant cells, were further analyzed. In Arabidopsis, NET1A is anchored at the plasma membrane and predominates at cell junctions, the plasmodesmata [31]. Besides, NET1A was also found to be involved in response to abscisic acid (ABA), the mitogen-activated protein kinase (MAPK) signaling pathway, and the calcium transduction pathway in upland cotton [32]. The two NET genes identified in this study might play an important role during fiber development. SI was also a crucial trait for cotton yield. The qtl19 was only associated with SI in six different environments. Within this QTL region, GH_A07G1389 encoded a tetratricopeptide repeat (TPR)-like superfamily protein, and the gene was a homologous gene of a reported gene that was responsible for the $\mathrm{Li}_{\mathrm{y}}$ short fiber phenotype. Silencing of the $L i_{y}$ gene could significantly reduce the fiber length in upland cotton [33]. GH_A07G1389 showed a high expression from -3 DPA to 1 DPA in fiber development, inferring that the differential expression of this gene in ovules might affect the fiber development of cotton. A large number of fiber development-related genes in the stable QTL interval were also found, such as GH_A08G0793 (SWEET12) [18] and GH_D09G1670 (EXPA4) [34], which might contribute to fiber yield improvement in breeding practice.

\section{Conclusions}

Mining the elite loci and alleles related to yield components has more practical value for genetic improvement of crop yield via molecular breeding by design. Here, the phenotyping data of four main yield components, boll number per plant (BN), boll weight (BW), lint percentage (LP) and seed index (SI), from 242 upland cotton accessions were continuously investigated under multiple field environments over the years. Genetic analysis indicated that LP had the highest heritability, following SI, BW, and BN. Through GWAS analysis, we identified 95 non-redundant and stable QTLs with 12, 23, 45, 33 for BN, BW, LP and SI, respectively. Of them, 15 QTLs were associated simultaneously with two or more traits and contributed pleiotropically cotton yield components, and 12 QTLs were detected repeatedly in more than six environments as high-confidence QTLs. Also, 10 pairs of homologous QTLs between $\mathrm{A}$ and $\mathrm{D}$ sub-genomes were detected. Further, we identified two NET genes (GH_A08G0716 and GH_A08G0783) located in a stable QTL hotspot (qtl24, qtl25 and qlt26) and predominately expressed in early fiber development stages. A gene encoding tetratricopeptide repeat (TPR)like superfamily protein (GH_A07G1389) in stable qtl19 region, were important candidate genes for improving the cotton yield. The present study might contribute novel elite loci and gene resources for yield improvement in cotton breeding practice.

\section{Methods \\ Plant Materials}

A natural population of 242 upland cotton accessions (Additional file 1 Table S1) was planted in 13 natural environments following local agronomic practices in China, including Korla, Xinxiang and Nanyang in 2011, 2012 and 2013, Shawan in 2016 and 2017, Yancheng and Dangtu in 2018 (Additional file 2 Table S2). All the accessions were planted with a random complete block design (RCBD) with two replicates in every environment and two rows in each replicate. In korla and Shawan, row length was $2 \mathrm{~m}$, with a $66+10 \mathrm{~cm}$ (wide/narrow) row distance and a $10 \mathrm{~cm}$ plant distance. Row length was $5 \mathrm{~m}$ in Xinxiang, and row distance was $80 \mathrm{~cm}$ with a $25 \mathrm{~cm}$ plant distance. In Nanyang, Yancheng and Dangtu, row length was $4.5 \mathrm{~m}$, row distance was $80 \mathrm{~cm}$ and plant distance was $33 \mathrm{~cm}$. All accessions were collected and preserved by Nanjing Agricultural University, China. All necessary permits for planting and investigating the set of the natural population were obtained from Nanjing Agricultural University, China.

\section{Phenotype investigation and data analysis}

Four yield-related traits BN, BW, LP and SI were measured in each environment to explore the phenotypic variation of cotton. At the mature stage of cotton, ten plants for each accession were selected randomly from the middle of each row. The BN was counted with ten biological replicates of each accession. The BW was weighed with 20 mature boll samples randomly harvested from the middle branches with 2 bolls per plant. After ginning, the LP was calculated based on the fraction of lint weight to seed-cotton weight, and the SI was weighed with 100 normally developed seeds.

In this study, the data for BW and LP from 13 natural environments, and BN and SI from 11 natural environments, were used for analysis. The best linear unbiased predictors (BLUPs) of the genotypic effects based on a mixed linear model for each trait were conducted by the lme4 package of $\mathrm{R}$ software [35]. The breeding value was evaluated by the best linear unbiased predictors adding population mean (marked as "BLUP") and used for correlation analysis and GWAS. The density distributions of phenotypic value were performed using "ggplot2" package in $\mathrm{R}$ software. ANOVA was conducted for 
phenotypic data in different environments using PROC GLM procedure in SAS software [36]. All variations from different sources were treated as random effects with a multi environments random blocks linear model. The statistical model was $y_{i j k}=\mu+\alpha_{i}+\beta_{j}+\gamma_{k j}+(\alpha \beta)$ ${ }_{\mathrm{ij}}+\varepsilon_{\mathrm{ijk}}$, that $\mu$ indicates the overall mean, $\alpha_{\mathrm{i}}$ indicates the genetic effect of the $i_{\text {th }}$ genotype, $\beta_{j}$ indicates the effect of the $j_{\text {th }}$ environment, $\gamma_{\mathrm{kj}}$ indicates the random effect of the $k_{\text {th }}$ replicate in the $j_{\text {th }}$ environment, $(\alpha \beta)_{i j}$ indicates the interaction effect between the $i_{\text {th }}$ genotype and the $j_{\text {th }}$ environment, $\varepsilon_{\mathrm{ijk}}$ indicates the residual [37]. The formula calculated the broad-sense heritability of each trait: $h^{2}=\sigma_{g}^{2} /\left(\sigma_{g}^{2}+\sigma_{g e}^{2}\left(n+\sigma_{\varepsilon}^{2} / r n\right)\right.$, that $\sigma_{g}^{2}$ indicates the genotype variance, $\sigma_{g e}$ indicates the genotype by environment interaction variance, $\sigma_{\varepsilon}^{2}$ indicates the error variance, $n$ indicates the number of environments, and $r$ indicates the number of replications [38]. Variance values used for broad-sense heritability were calculated using the REML method for the SAS VARCOMP procedure in SAS software. The correlation coefficient and the significance between traits were conducted by SPSS 22.0 software.

\section{SNP genotyping}

Genomic DNA of young leaf tissues of each accession was extracted using the CTAB method as described by Paterson et al. (1993) [39]. The 242 accessions were genotyped by the CottonSNP80K array, which contained 77,774 SNPs [16]. After filtering the SNPs with a calling rate of $<0.9$ and $\mathrm{MAF}<0.05$, high-quality SNPs were obtained. The probe sequences of the high-quality SNPs were mapped to the TM-1 V2.1 genome [11] to determine the exact physical location. When a probe sequence is mapped to multiple loci, the corresponding SNP was discarded. Finally, a dataset that contained 56,010 unique mapped high-quality SNPs was used for GWAS analysis.

\section{GWAS analysis}

Based on a multi-locus random-SNP-effect mixed linear model (mrMLM) [22], six methods ("mrMLM", "FASTmrMLM", "FASTmrEMMA", "pKWmEB", "ISIS EMBLASSO" and "pLARmEB") in the R package "mrMLM" were used to identify QTNs for each trait. The parameters of program were set as: Critical $P$-value $=0.001$; Search radius of candidate gene $(\mathrm{Kb})=100$; Critical LOD score $=3$. Also, we selected $Q+K$ statistical model for GWAS analysis. A kinship (K) matrix was obtained directly in the "mrMLM" program. According to our previous study [40], $\mathrm{k}=3$ was selected, and a population structure $(\mathrm{Q})$ matrix was calculated by admixture version 1.3. The 52 data sets, including the phenotyping data from the different environments and the BLUP for each trait, were used for the GWAS analysis.

\section{Identification of QTLs and candidate genes}

Due to the uneven distribution of the genotyped SNPs and referenced in the previous report [19], we selected the lowest LD $(200 \mathrm{~kb})$ of the chromosome as a threshold to merge the adjacent QTNs into the same QTL. According to a self-written shell script, candidate genes were obtained from the QTL regions on the reference genome. To determine which genes are related to cotton yield components, the transcriptome FastQ data of TM-1 tissues, including root, stem, leaf, petal, torus, sepal, bract, anther, filament, pistil, ovule and fiber tissues at $-3,0,1,3,5$ days post-anthesis (DPA), ovules at $10,15,20$, and $25 \mathrm{DPA}$, and fiber tissues at 10, 15, 20, and 25 DPA, were downloaded from NCBI Sequence Read Archive collection PRJNA490626 [11]. Using the mapping software Hisat2 [41], uniquely mapped reads were mapped to the reference genome and counted for all cotton annotated genes using HTSeq-Count [42]. The following parameters were used for the counting process: -f bam, -r name, -s no, -m union, -a 20. These count files were used for the identification of DEGs using the program edgeR [43]. A candidate gene was considered stable when the TPM (transcripts per million) was greater than 3 and was used for further analysis. AgriGO V2.0, an online bioinformatics tool, was used to analyze the candidate genes [44].

\section{Abbreviations \\ ANOVA: Analysis of variance; BLUPs: The best linear unbiased predictors; BLUP: The best linear unbiased prediction; BN: Boll number; GO: Gene ontology; GWAS: Genome-wide association studies; SI: Seed index; LD: Linkage disequi- librium; LP: Lint percentage; MAF: Minor allele frequency; QTLs: Quantitative trait loci; QTNs: Quantitative trait nucleotides; BW: Boll weight; SNP: Single nucleotide polymorphism}

\section{Supplementary Information}

The online version contains supplementary material available at https://doi. org/10.1186/s12870-021-03009-2.

Additional file 1: Table S1. Information of 242 upland cotton accessions used in this study.

Additional file 2: Table S2. Phenotypic variation for four yield-related traits in multiple field environments and BLUP.

Additional file 3: Figure S1. Density distributions of four yield-related traits in upland cotton natural population.

Additional file 4: Table S3. Information on high quality SNPs genotyped by CottonSNP80K array.

Additional file 5: Table S4. QTNs and QTLs of boll number per plant, boll weight, lint percentage and seed index detected by multi-loci MLM model.

Additional file 6: Table S5. The candidate QTLS, co-located QTLs and high-confidence QTLs identified for the four yield-related traits.

Additional file 7: Table S6. Information of homologous QTLs for the four yield-related traits.

Additional file 8: Figure S2. The number of candidate QTLs associated with the yield-related traits on At and Dt sub-genomes. 
Additional file 9: Figure S3. Venn diagram of QTLs associated with four yield-related traits.

Additional file 10: Table S7. Expression profile of candidate genes from the QTL regions related to the four yield-related traits.

Additional file 11: Table S8. Function analysis of genes in QTL regions related to the four yield-related traits.

Additional file 12: Figure S4. Candidate genes related to the qlt19 associated with $\mathrm{SI}$ and located on chromosome A07. a. Expression heatmap of candidate genes in qlt19. b. The expression pattern of GH_A07G1389 in different tissues. c. Box plots for the phenotypic values of QTN closest to GH_A07G1389.

\section{Acknowledgements}

We thank the members and collaborators of Cotton Germplasm Enhancement and Application Engineering Research Center (Ministry of Education) in Nanjing Agricultural University for continuous phenotypic investigation of the set of the natural population for years and environments. Thanks to Dr. Muhammad Waqas Amjid from College of Agriculture in Nanjing Agricultural University for helpful editing and comments. Many thanks to the high-performance computing platform of Bioinformatics Center in Nanjing Agricultural University for providing data analysis facilities and the National Medium-term Gene Bank of Cotton in China for providing some landraces seeds.

\section{Authors' contributions}

Experiments were designed by WZG. Experiments were performed by GZZ, XHS, SH, XW, WW, and QJC. GZZ and SH drafted the manuscript, WZG revised the manuscript. All authors read and approved the final manuscript.

\section{Funding}

This program was financially supported in part by the National Key R \& D Program for Crop Breeding (2018YFD0100400), the Fundamental Research Funds for the Central Universities (KYYJ201901; KYZZ201904; KYYJ202007), the open funds of the State Key Laboratory of Crop Genetics and Germplasm Enhancement (ZW202007), Jiangsu Collaborative Innovation Center for Modern Crop Production project (No.10). The funders were not involved in the experimental design of the study, data collection, analysis and interpretation, and in writing the manuscript.

\section{Availability of data and materials}

RNA-Seq data in this study have been deposited at the National Center of Biotechnology Information (NCBI, http://www.ncbi.nlm.nih.gov/) under the accessions PRJNA490626.

\section{Declarations}

Ethics approval and consent to participate Not applicable.

\section{Consent for publication}

Not applicable.

\section{Competing interests}

The authors declared that they had no competing interests.

\section{Author details}

${ }^{1}$ State Key Laboratory of Crop Genetics and Germplasm Enhancement, Cotton Germplasm Enhancement and Application Engineering Research Center (Ministry of Education), Nanjing Agricultural University, Nanjing 210095, China. ${ }^{2}$ Institute of Agricultural Sciences in Coastal Area of Jiangsu Province, Yancheng 224002, China. ${ }^{3}$ Engineering Research Center for Cotton (the Ministry of Education), Xinjiang Agricultural University, Urumqi 830052, China.

Received: 14 December 2020 Accepted: 5 May 2021

Published online: 31 May 2021

\section{References}

1. Chen ZJ, Scheffler BE, Dennis E, Triplett BA, Zhang T, Guo W, Chen X, Stelly DM, Rabinowicz PD, Town CD, et al. Toward sequencing cotton (Gossypium) genomes. Plant Physiol. 2007;145(4):1303-10.

2. Zhang HB, Li Y, Wang B, Chee PW. Recent advances in cotton genomics. Int J Plant Genom. 2008;2008:742304.

3. Qin H, Guo W, Zhang YM, Zhang T. QTL mapping of yield and fiber traits based on a four-way cross population in Gossypium hirsutum L. Theor Appl Genet. 2008;117(6):883-94.

4. Shen XL, Guo WZ, Lu QX, Zhu XF, Yuan YL, Zhang TZ. Genetic mapping of quantitative trait loci for fiber quality and yield trait by RIL approach in Upland cotton. Euphytica. 2007;155(3):371-80.

5. Liu RZ, Wang BH, Guo WZ, Qin YS, Wang LG, Zhang YM, Zhang TZ. Quantitative trait loci mapping for yield and its components by using two immortalized populations of a heterotic hybrid in Gossypium hirsutum L. Mol Breed. 2012;29(2):297-311.

6. Zhang T, Hu Y, Jiang W, Fang L, Guan X, Chen J, Zhang J, Saski CA, Scheffler BE, Stelly DM, et al. Sequencing of allotetraploid cotton (Gossypium hirsutum L. acc. TM-1) provides a resource for fiber improvement. Nat Biotechnol. 2015;33(5):531-7.

7. Li FG, Fan GY, Lu CR, Xiao GH, Zou CS, Kohel RJ, Ma ZY, Shang HH, Ma XF, Wu JY, et al. Genome sequence of cultivated Upland cotton (Gossypium hirsutum TM-1) provides insights into genome evolution. Nature Biotechnol. 2015;33(5):524-30.

8. Yuan DJ, Tang ZH, Wang MJ, Gao WH, Tu LL, Jin X, Chen LL, He YH, Zhang $L$, Zhu LF, et al. The genome sequence of Sea-Island cotton (Gossypium barbadense) provides insights into the allopolyploidization and development of superior spinnable fibres. Sci Rep. 2015;5:17662.

9. Liu X, Zhao B, Zheng HJ, Hu Y, Lu G, Yang CQ, Chen JD, Chen JJ, Chen DY, Zhang $L$, et al. Gossypium barbadense genome sequence provides insight into the evolution of extra-long staple fiber and specialized metabolites. Sci Rep. 2015;5:14139.

10. Wang M, Tu L, Yuan D, Zhu D, Shen C, Li J, Liu F, Pei L, Wang P, Zhao G, et al. Reference genome sequences of two cultivated allotetraploid cottons, Gossypium hirsutum and Gossypium barbadense. Nat Genet. 2019;51(2):224-9.

11. Hu Y, Chen JD, Fang L, Zhang ZY, Ma W, Niu YC, Ju LZ, Deng JQ, Zhao T, Lian JM, et al. Gossypium barbadense and Gossypium hirsutum genomes provide insights into the origin and evolution of allotetraploid cotton. Nat Genet. 2019;51(4):739-48.

12. Song C, Li W, Pei X, Liu Y, Ren Z, He K, Zhang F, Sun K, Zhou X, Ma X, et al. Dissection of the genetic variation and candidate genes of lint percentage by a genome-wide association study in upland cotton. Theor Appl Genet. 2019;132(7):1991-2002.

13. Fang L, Wang Q, Hu Y, Jia Y, Chen J, Liu B, Zhang Z, Guan X, Chen S, Zhou $B$, et al. Genomic analyses in cotton identify signatures of selection and loci associated with fiber quality and yield traits. Nat Genet. 2017;49(7):1089-98.

14. Wang M, Tu L, Lin M, Lin Z, Wang P, Yang Q, Ye Z, Shen C, Li J, Zhang L, et al. Asymmetric subgenome selection and cis-regulatory divergence during cotton domestication. Nat Genet. 2017;49(4):579-87.

15. Ma Z, He S, Wang X, Sun J, Zhang Y, Zhang G, Wu L, Li Z, Liu Z, Sun $G$, et al. Resequencing a core collection of upland cotton identifies genomic variation and loci influencing fiber quality and yield. Nat Genet. 2018;50(6):803-13.

16. Huang C, Nie X, Shen C, You C, Li W, Zhao W, Zhang X, Lin Z. Population structure and genetic basis of the agronomic traits of upland cotton in China revealed by a genome-wide association study using high-density SNPs. Plant Biotechnol J. 2017;15(11):1374-86.

17. Cai C, Guozhong Z, Tianzhen Z, Wangzhen G. High-density 80 K SNP array is a powerful tool for genotyping G. hirsutum accessions and genome analysis. BMC Genom. 2017;18(1):654.

18. Wang R, Liu M, Yuan M, Oses-Prieto JA, Cai X, Sun Y, Burlingame AL, Wang $Z Y$, Tang W. The brassinosteroid-activated BRI1 receptor kinase is switched off by dephosphorylation mediated by cytoplasm-localized PP2A B' subunits. Mol Plant. 2016;9(1):148-57.

19. Zhang ZY, Ruan YL, Zhou N, Wang F, Guan XY, Fang L, Shang XG, Guo WZ, Zhu SJ, Zhang TZ. Suppressing a putative sterol carrier gene reduces plasmodesmal permeability and activates sucrose transporter genes during cotton fiber elongation. Plant Cell. 2017;29(8):2027-46. 
20. Zhu G, Gao W, Song X, Sun F, Hou S, Liu N, Huang Y, Zhang D, Ni Z, Chen $Q$, et al. Genome-wide association reveals genetic variation of lint yield components under salty field conditions in cotton (Gossypium hirsutum L.). BMC Plant Biol. 2020;20(1):23.

21. Su J, Fan S, Li L, Wei H, Wang C, Wang H, Song M, Zhang C, Gu L, Zhao S, et al. Detection of favorable QTL alleles and candidate genes for lint percentage by gwas in Chinese upland cotton. Front Plant Sci. 2016;7:1576.

22. Sun Z, Wang X, Liu Z, Gu Q, Zhang Y, Li Z, Ke H, Yang J, Wu J, Wu L, et al. A genome-wide association study uncovers novel genomic regions and candidate genes of yield-related traits in upland cotton. Theor Appl Genet. 2018;131(11):2413-25.

23. Wang SB, Feng JY, Ren WL, Huang B, Zhou L, Wen YJ, Zhang J, Dunwell $J M, X u$ S, Zhang YM. Improving power and accuracy of genome-wide association studies via a multi-locus mixed linear model methodology. Sci Rep. 2016;6:19444.

24. Ruan YL. Recent advances in understanding cotton fibre and seed development. Seed Sci Res. 2005;15(4):269-80.

25. Ruan YL. Boosting seed development as a new strategy to increase cotton fiber yield and quality. J Integr Plant Biol. 2013;55(7):572-5.

26. Applequist WL, Cronn R, Wendel JF. Comparative development of fiber in wild and cultivated cotton. Evol Dev. 2001;3(1):3-17.

27. Pugh DA, Offler CE, Talbot MJ, Ruan YL. Evidence for the role of transfer cells in the evolutionary increase in seed and fiber biomass yield in cotton. Mol Plant. 2010;3(6):1075-86.

28. Paterson AH, Saranga Y, Menz M, Jiang CX, Wright RJ. QTL analysis of genotype $x$ environment interactions affecting cotton fiber quality. Theor Appl Genet. 2003;106(3):384-96.

29. Zhang Z, Shang H, Shi Y, Huang L, Li J, Ge Q, Gong J, Liu A, Chen T, Wang $D$, et al. Construction of a high-density genetic map by specific locus amplified fragment sequencing (SLAF-seq) and its application to Quantitative Trait Loci (QTL) analysis for boll weight in upland cotton (Gossypium hirsutum.). BMC Plant Biol. 2016;16:79.

30. Nazir MF, Jia Y, Ahmed H, He S, labal MS, Sarfraz Z, Ali M, Feng C, Raza I, Sun $\mathrm{G}$, et al. Genomic insight into differentiation and selection sweeps in the improvement of upland cotton. Plants. 2020;9(6):711.

31. Deeks MJ, Calcutt JR, Ingle EKS, Hawkins TJ, Chapman S, Richardson AC, Mentlak DA, Dixon MR, Cartwright F, Smertenko AP, et al. A superfamily of actin-binding proteins at the actin-membrane nexus of higher plants. Curr Biol. 2012;22(17):1595-600.

32. Li HM, Liu SD, Ge CW, Zhang XM, Zhang SP, Chen J, Shen Q, Ju FY, Yang YF, Li Y, et al. Analysis of drought tolerance and associated traits in upland cotton at the seedling stage. Int J Mol Sci. 2019;20(16):3888.
33. Fang DD, Naoumkina M, Thyssen GN, Bechere E, Li P, Florane CB. An EMS-induced mutation in a tetratricopeptide repeat-like superfamily protein gene (Ghir_A12G008870) on chromosome A12 is responsible for the li(y) short fiber phenotype in cotton. Theor Appl Genet. 2020;133(1):271-82.

34. Lv LM, Zuo DY, Wang XF, Cheng HL, Zhang YP, Wang QL, Song GL, Ma ZY. Genome-wide identification of the expansin gene family reveals that expansin genes are involved in fibre cell growth in cotton. BMC Plant Biol. 2020;20(1):223.

35. Bates D, Machler M, Bolker BM, Walker SC. Fitting linear mixed-effects models using Ime4. J Stat Softw. 2015;67(1):1-48.

36. SAS I. Base SAS 9.4 procedures guide: statistical procedures. Cary, NC, USA: SAS Institute Inc, 2013.

37. Li XN, Zhang XL, Zhu LM, Bu YP, Wang XF, et al. Genome-wide association study of four yield-related traits at the R6 stage in soybean. BMC Genet. 2019;20(1):39

38. Piepho HP, Mohring J. Computing heritability and selection response from unbalanced plant breeding trials. Genetics. 2007;177:1881-8.

39. Paterson AH, Brubaker CL, Wendel JF. A rapid method for extraction of cotton (Gossypium spp.) genomic DNA suitable for RFLP or PCR analysis. Plant Mol Biol Rep. 1993;11(2):122-7.

40. Zhu G, Li W, Wang G, Li L, Si Q, Cai C, Guo W. Genetic basis of fiber improvement and decreased stress tolerance in cultivated versus semidomesticated upland cotton. Front Plant Sci. 2019;10:1572.

41. Pertea M, Kim D, Pertea GM, Leek JT, Salzberg SL. Transcript-level expression analysis of RNA-seq experiments with HISAT StringTie and Ballgown. Nat Protoc. 2016;11(9):1650-67.

42. Anders S, Pyl PT, Huber W. HTSeq-a Python framework to work with highthroughput sequencing data. Bioinformatics. 2015;31(2):166-9.

43. Robinson MD, McCarthy DJ, Smyth GK. edgeR: a Bioconductor package for differential expression analysis of digital gene expression data. Bioinformatics. 2010;26(1):139-40.

44. Tian T, Liu Y, Yan H, You Q, Yi X, Du Z, Xu W, Su Z. agriGO v2.0: a GO analysis toolkit for the agricultural community, 2017 update. Nucleic Acids Res. 2017;45(W1):W122-9.

\section{Publisher's note}

Springer Nature remains neutral with regard to jurisdictional claims in published maps and institutional affiliations.
Ready to submit your research? Choose BMC and benefit from:

- fast, convenient online submission

- thorough peer review by experienced researchers in your field

- rapid publication on acceptance

- support for research data, including large and complex data types

- gold Open Access which fosters wider collaboration and increased citations

- maximum visibility for your research: over $100 \mathrm{M}$ website views per year

At BMC, research is always in progress.

Learn more biomedcentral.com/submissions 